\title{
A Case of Staphylococcus Associated Glomerulonephritis Confused with Acute Interstitial Nephritis
}

\author{
Chathri Ratnayake, Ken Teo \\ Department of Internal Medicine, Eastern Health, Melbourne, Australia \\ Email: chathri.r@gmail.com
}

How to cite this paper: Ratnayake, C. and Teo, K. (2020) A Case of Staphylococcus Associated Glomerulonephritis Confused with Acute Interstitial Nephritis. Case Reports in Clinical Medicine, 9, 263-268. https://doi.org/10.4236/crcm.2020.99037

Received: July 31, 2020

Accepted: September 5, 2020

Published: September 8, 2020

Copyright $\odot 2020$ by author(s) and Scientific Research Publishing Inc. This work is licensed under the Creative Commons Attribution International License (CC BY 4.0).

http://creativecommons.org/licenses/by/4.0/

\section{(c) (i) Open Access}

\begin{abstract}
Staphylococcus associated glomerulonephritis (SAGN) is an uncommon infection-related glomerulonephritis. Although infection is typically ongoing when patients present with SAGN, in the case of deep-seated infections, the presence of infection may not be apparent. The aim of this study is to highlight the importance of renal biopsy when the diagnosis is not obvious. We present a case of staphylococcus associated glomerulonephritis in a patient treated with beta-lactam antibiotics for staphylococcus bacteraemia. A renal biopsy helped differentiate between interstitial nephritis and staphylococcus associated glomerulonephritis.
\end{abstract}

\section{Keywords}

Staphylococcus Associated Glomerulonephritis, Acute Interstitial Nephritis, Nomenclature, Renal Biopsy

\section{Introduction}

Acute kidney injury in the setting of infection can be multifactorial and difficult to differentiate. Although infection-related glomerulonephritis is a known cause, it is uncommon with a prevalence of $0.8 \%$ [1]. Staphylococcus associated glomerulonephritis is on the rise due to change in patient demographics and increasing incidence of community acquired methicillin resistant Staphylococcus aureus. SAGN mainly affects older adult males and its incidence peaks between the fifth and seventh decades of life [1] [2]. Prevalence of drug induced interstitial nephritis is also on the rise due to widespread use of antibiotics [3]. True incidence of these pathologies is likely underestimated as confirmatory renal biopsy is often not performed in older frail patients. Challengingly, this is the same 
population in whom these pathologies are most commonly seen. The aim of this paper is to demonstrate the importance of histological diagnosis of renal injury to rule out other nephritides that can have similar clinical presentations but have different treatment options and prognosis.

\section{Case Report}

A 64-year-old man presented to emergency department (ED) with painful left arm at the site of a previous intravenous (IV) cannulation which was inserted during a recent hospital admission for melaena. His prior medical history was significant for severe dilated ischemic cardiomyopathy, atrial fibrillation and diet-controlled type II Diabetes mellitus. He had an implantable cardioverter defibrillator in situ for primary prevention of malignant arrythmias.

He was diagnosed with superficial thrombophlebitis and discharged from ED on the same day with instruction to use topical anti-inflammatory for symptom relief. He presented a week later, with lethargy and feeling generally unwell. $\mathrm{He}$ became febrile and hypotensive in ED. Blood cultures and other basic pathology were completed. He was then started on IV Piperacillin-tazobactam, Vancomycin and Flucloxacillin for presumed IV line related sepsis. Four of his initial blood cultures became positive for methicillin sensitive Staphylococcus aureus. IV Flucloxacillin was continued, other antibiotics were ceased. Trans-thoracic echocardiogram did not show vegetations, due to restrictions in the COVID pandemic, he did not get a transoesophageal echocardiogram. Total antibiotic duration was decided as 6 weeks to treat presumed infective endocarditis due to prolonged bacteraemia, presence of implantable cardioverter and absence of definitive imaging. He remained stable and was discharged from hospital on day 5 of Flucloxacillin.

He was referred back to emergency department on day 8 of Flucloxacillin, when his routine biochemistry showed an acute worsening of renal function. His creatinine was elevated to $462 \mu \mathrm{mol} / \mathrm{L}$ from baseline $143 \mu \mathrm{mol} / \mathrm{L}$. His estimated glomerular filtration rate had dropped from 45 to $11 \mathrm{~mL} / \mathrm{min} / 1.73 \mathrm{~m}^{2}$. A differential diagnosis of beta-lactam induced acute interstitial nephritis (AIN) was suspected and Flucloxacillin was changed to Vancomycin.

He had an active urine sediment with more than $1000 \mathrm{red}$ cells/hpf. There were no casts. He had no proteinuria. Tests showed white cell count $6.9 \times 109 / 1$ (N $4-10 \times 109 / 1)$, eosinophils $0.15 \times 109 / 1(\mathrm{~N} 0.02-0.5 \times 109 / 1)$ and albumin 22 g/L. Complement (C) levels were significant for low C3 $0.29(0.9-1.8)$ and normal C4. Antinuclear antibody (ANA), anti-double stranded DNA antibody (Anti-dsDNA) and antineutrophil cytoplasmic antibodies (ANCA) were negative. Renal tract imaging was normal. On day 5 of his admission he had a renal biopsy. His biopsy showed proliferative and exudative glomerulonephritis with subepithelial IgG and C3 deposits in keeping with "post-infection glomerulonephritis" (Figure 1 and Figure 2). There were also very focal IgA deposits in the mesangium. His antibiotics were changed to beta-lactam agent IV Cefazolin. 


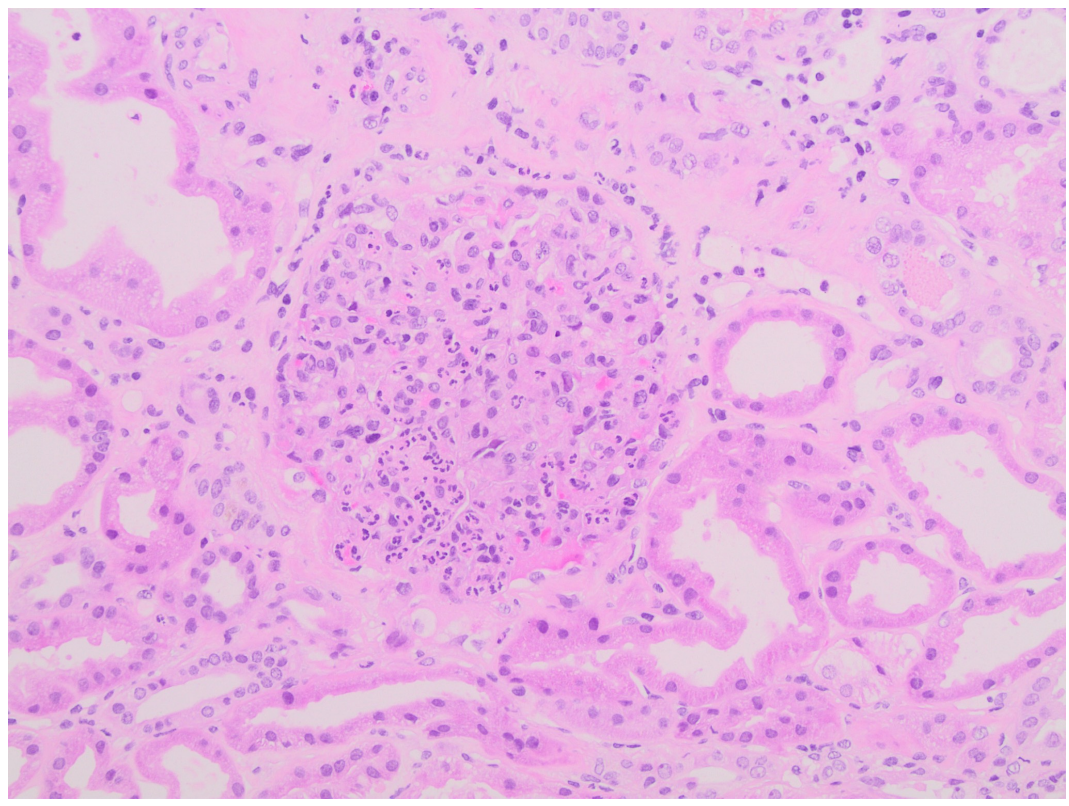

Figure 1. Glomerulus showing global exudation of neutrophils (200× magnification).

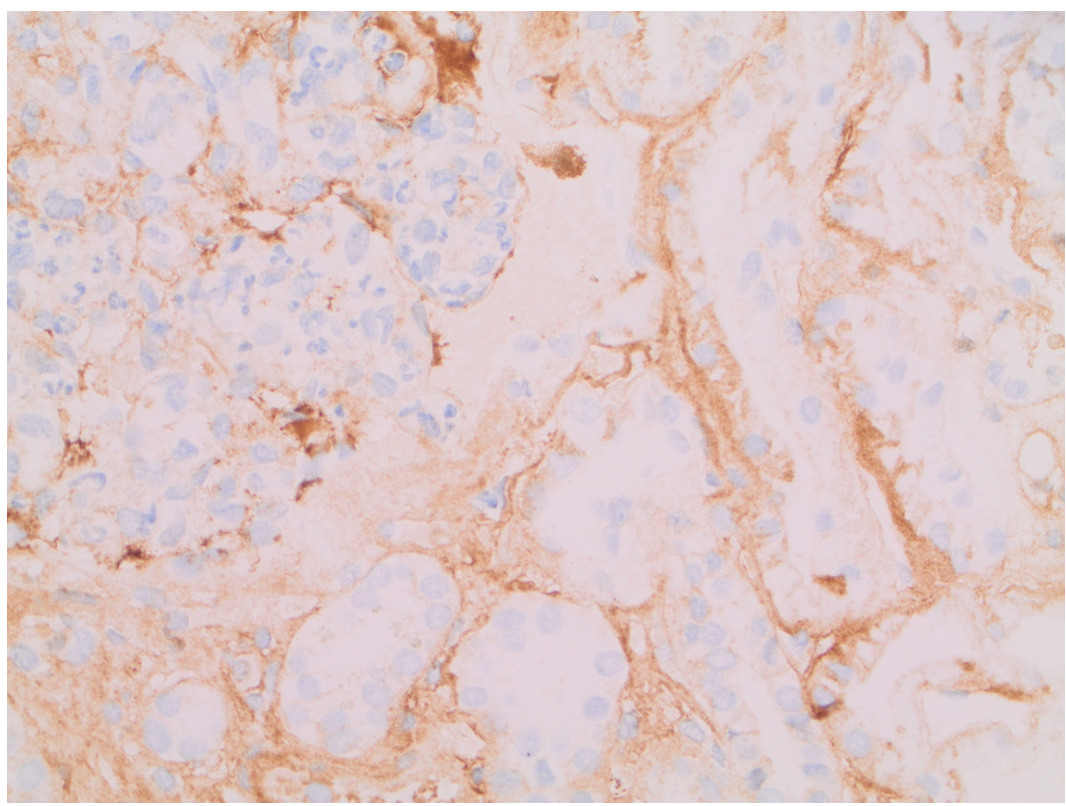

Figure 2. Immunoperoxidase stains showing focal positive subepithelial "humps" and finely granular mesangial staining (400× magnification).

His renal function improved and was discharged from hospital to complete total of 6 weeks of antibiotics. He recovered well and was followed up with weekly renal function assessment. His renal function returned to baseline at the end of antibiotic therapy.

\section{Discussion}

Infection associated glomerulonephritis (GN) is one of the oldest renal disease entities described in literature. Any glomerular pathology that result from an in- 
fection can be considered as infection associated glomerulonephritis. However, this terminology encompasses a few different glomerulonephritides which makes it unclear and confusing at times [4]. Infection associated GN can be broadly divided to post infection glomerulonephritis (PIGN) and glomerulonephritis with active ongoing infection. Post streptococcus glomerulonephritis is the posterchild of PIGN and the only well documented cause of PIGN. In the case of PIGN, infection is followed by a latent period of days to weeks, during which the patient returns to his or her usual state of health before GN occurs. Staphylococcus infection associated GN (SAGN) is the prototype of GN that occurs with a concurrent infection [1].

These two entities, although grouped under one blanket term of infection associated glomerulonephritis, are different in their pathogenesis, treatment and prognosis. Therefore, it is important to define the pathology using the correct nomenclature to reflect the timing of GN in relation to infection. In the case described above, this patient had glomerulonephritis before completing minimum duration of treatment for staphylococcus bacteraemia [5]. Therefore, he had SAGN, not PIGN as biopsy report reads. This reflects the need for medical professionals to have a better understanding of the classification and terminology of glomerulonephritis.

SAGN is uncommon with an incidence of $0.6 \%-0.8 \%$ [2] [6] but it is on the rise due to emerging drug-resistant strains of staphylococcus, increasing the elderly population with comorbidities, and intravenous drug addiction. In this case, acute interstitial nephritis was the initial working diagnosis. This is due to a reasonable suspicion of penicillin induced AIN, supported by its higher prevalence of $15 \%-27 \%$ and timing of the kidney injury [7] [8]. In our patient, the renal function was impaired, but given that it remained stable for five days there was deliberation for renal biopsy. Confirmatory diagnosis of SAGN from renal biopsy allowed continuous use of beta-lactam antibiotics in this patient. This also prevented mislabelling this patient with a $\beta$-lactam allergy which would have restricted future antibiotic choice and resulted in inappropriate prescribing [9]. It is important to be mindful of risks of renal biopsy and ensure benefits of renal biopsy outweigh the risks. This patient had a perinephric haematoma post-biopsy which resolved without requiring any intervention.

No single pathological feature is pathognomonic for SAGN. Diagnosis of SAGN should be suspected in a patient with evidence of staphylococcus infection concurrent with the onset of glomerulonephritis if they also have hypocomplementemia, proliferative glomerulonephritis with C3 dominant or codominant with IgG/IgA deposits and subepithelial humps on electron microscopy [6] [10] [11].

Case reports of SAGN in recent times have described dominant mesangial IgA deposition [12]. For this reason, SAGN is now often commonly referred to as IgA-dominant infection-associated GN. However, there is emerging evidence to suggest that there is a spectrum of IgA staining. Satoskar et al. showed that IgA staining can vary from trace or negative to strong, with the majority of the biop- 
sies showing weak to moderate staining. This is in keeping with our patient's biopsy which showed weak IgA staining [2].

Our patient's renal function improved at the end of the 6-week course of intravenous antibiotics. This is in keeping with usual course of SAGN where successful eradication of infection results in resolution of glomerulonephritis.

\section{Conclusion}

A renal biopsy should be performed if staphylococcus associated glomerulonephritis is suspected as a wide range of pathologies could present with the same clinical picture and differentiation of these syndromes is important for treatment and prognostication.

\section{Acknowledgements}

Authors express sincere gratitude to the patient for permission to publish the case report. We also thank pathologist Dr. Metta MacNeil for providing histology slides.

\section{Conflicts of Interest}

The authors declare no conflicts of interest regarding the publication of this paper.

\section{References}

[1] Satoskar, A.A., Parikh, S.V. and Nadasdy, T. (2020) Epidemiology, Pathogenesis, Treatment and Outcomes of Infection-Associated Glomerulonephritis. Nature Reviews Nephrology, 16, 32-50. https://doi.org/10.1038/s41581-019-0178-8

[2] Satoskar, A.A., Suleiman, S., Ayoub, I., Hemminger, J., Parikh, S., Brodsky, S.V., et al. (2017) Staphylococcus Infection-Associated GN-Spectrum of IgA Staining and Prevalence of ANCA in a Single-Center Cohort. Clinical Journal of the American Society of Nephrology, 12, 39-49. https://doi.org/10.2215/CJN.05070516

[3] Goicoechea, M., Rivera, F. and López-Gómez, J.M. (2013) Increased Prevalence of Acute Tubulointerstitial Nephritis. Nephrology Dialysis Transplantation, 28, 112-115. https://doi.org/10.1093/ndt/gfs143

[4] Glassock, R.J., Alvarado, A., Prosek, J., Hebert, C., Parikh, S., Satoskar, A., et al. (2015) Staphylococcus-Related Glomerulonephritis and Poststreptococcal Glomerulonephritis: Why Defining "Post" Is Important in Understanding and Treating Infection-Related Glomerulonephritis. American Journal of Kidney Diseases, 65, 826-832. https://doi.org/10.1053/j.ajkd.2015.01.023

[5] Holland, T.L., Arnold, C. and Fowler Jr., V.G. (2014) Clinical Management of Staphylococcus aureus Bacteremia: A Review. JAMA, 312, 1330-1341. https://doi.org/10.1001/jama.2014.9743

[6] Nasr, S.H., Markowitz, G.S., Stokes, M.B., Said, S.M., Valeri, A.M. and D'Agati, V.D. (2008) Acute Postinfectious Glomerulonephritis in the Modern Era: Experience with 86 Adults and Review of the Literature. Medicine (Baltimore), 87, 21-32. https://doi.org/10.1097/md.0b013e318161b0fc

[7] Haas, M., Spargo, B.H., Wit, E.J. and Meehan, S.M. (2000) Etiologies and Outcome of Acute Renal Insufficiency in Older Adults: A Renal Biopsy Study of 259 Cases. 
American Journal of Kidney Diseases, 35, 433-447.

https://doi.org/10.1016/S0272-6386(00)70196-X

[8] Farrington, K., Levison, D.A., Greenwood, R.N., Cattell, W.R. and Baker, L.R. (1989) Renal Biopsy in Patients with Unexplained Renal Impairment and Normal Kidney Size. Q JMed, 70, 221-233

[9] Lazarus, B., Davies, M.R.P., Trubiano, J.A. and Pellicano, R. (2020) Time to Acute Kidney Injury in $\beta$-Lactam-Induced Acute Interstitial Nephritis. Kidney International Reports, 5, 1068-1070. https://doi.org/10.1016/j.ekir.2020.04.008

[10] Nasr, S.H., Radhakrishnan, J. and D’Agati, V.D. (2013) Bacterial Infection-Related Glomerulonephritis in Adults. Kidney International, 83, 792-803.

https://doi.org/10.1038/ki.2012.407

[11] Nasr, S.H., Fidler, M.E., Valeri, A.M., Cornell, L.D., Sethi, S., Zoller, A., et al. (2011) Postinfectious Glomerulonephritis in the Elderly. Journal of the American Society of Nephrology, 22, 187-195. https://doi.org/10.1681/ASN.2010060611

[12] Wehbe, E., Salem, C., Simon, J.F., Navaneethan, S.D. and Pohl, M. (2011) IgA-Dominant Staphylococcus Infection-Associated Glomerulonephritis: Case Reports and Review of the Literature. NDT Plus, 4, 181-185.

https://doi.org/10.1093/ndtplus/sfr017 